12,05

\title{
Мессбауэровские исследования композитов гидроксиапатит/феррооксиды
}

\author{
(C) A.C. Камзин ${ }^{1}$, N. Wakiya ${ }^{2,3}$ \\ ${ }^{1}$ Физико-технический институт им. А.Ф. Иофрфе РАН, \\ Санкт-Петербург, Россия \\ ${ }^{2}$ Department of Electronics and Materials Science, Shizuoka University, \\ Naka-ku, Hamamatsu, Japan \\ ${ }^{3}$ Research Institute of Electronics, Shizuoka University, \\ 3-5-1 Johoku, Naka-ku, Hamamatsu, Japan \\ E-mail: Kamzin@mail.ioffe.ru \\ (Поступила в Редакцию 10 мая 2018 г.)
}

Исследованы магнитные композитные (МК) частицы, состоящих из гидроксиапатита и оксидов железа (HAp/FeOxid), синтезированных как при температурах пиролиза при синтезе МК: 800, 900 и $1000^{\circ} \mathrm{C}$, так и при различных концентрациях феррооксидов в композите HAp:FeOxid, а именно, 1:3, 1:2 и 1:1 (при температуре пиролиза $1000^{\circ} \mathrm{C}$ ). Установлено, что МК HAp/FeOxid образованы матрицей из гидроксиапатита, обеспечивающей биологическую совместимость МК, в которой находятся частицы оксидов железа. Мессбауэровскими исследованиями показано, что в синтезированных МК НАp/FeOxid одновременно наблюдаются фазы маггемита $\left(\gamma-\mathrm{Fe}_{2} \mathrm{O}_{3}\right)$, магнетита $\left(\mathrm{Fe}_{3} \mathrm{O}_{4}\right), \varepsilon-\mathrm{Fe}_{2} \mathrm{O}_{3}$ и акаганита $(\beta$ - $\mathrm{FeOH})$. Компонента $\varepsilon-\mathrm{Fe}_{2} \mathrm{O}_{3}$, обладающая гигантской магнитной анизотропией, составляет в МК HAp/FeOxid до $\sim 40 \%$ от оксидов железа (FeOxid), что делает полученные МК весьма перспективными для различных применений в том числе и биомедицинских.

DOI: $10.21883 /$ FTT.2018.12.46772.121

\section{1. Введение}

Сочетание магнитных свойств с наноразмерными и поверхностными эффектами таких известных полиморфных модификаций оксидов железа таких, как магнетит $\left(\mathrm{Fe}_{3} \mathrm{O}_{4}\right)$, гематит $\left(\alpha-\mathrm{Fe}_{2} \mathrm{O}_{3}\right)$, маггемит $\left(\gamma-\mathrm{Fe}_{2} \mathrm{O}_{3}\right)$ и феррооксид $\varepsilon$ - $\mathrm{Fe}_{2} \mathrm{O}_{3}$ привлекают большое внимание как с точки зрения изучения фундаментальных явлений, так и в плане новых приложений, например, для биомедицины в качестве усилителей контрастности снимков магнитно-резонансной томографии, для магнитной гипертермической терапии злокачественных опухолей, целевой доставки лекарственных средств [1-5]. Магнитная гипертермическая терапия (МГТ) - это способ лечения злокачественных опухолей, путем нагревания расположенных в опухоли магнитных частиц внешним переменным магнитным полем до температур $\sim 42-45^{\circ} \mathrm{C}$, при которых происходит некроз злокачественных клеток, тогда как здоровые клетки при этих температурах не повреждаются. Метод МГТ чрезвычайно привлекателен из-за безопасности лечения, небольших физических или психических напряжений для пациентов в отличии, например от лучевой терапии.

В плане применения для медицины активно изучались встречающиеся в природе и существующие как в объемных, так и в наноразмерных формах оксиды железа, а именно: маггемит $\left(\gamma-\mathrm{Fe}_{2} \mathrm{O}_{3}\right)[5,6]$, магнетит $\left(\mathrm{Fe}_{3} \mathrm{O}_{4}\right)$ [7]. Эти оксиды легко получаются множеством разновидностей методов синтеза МНЧ с различными морфологиями, размерами и распределением по размерам. Однако, магнитные характеристики маггемита и магнетита невысоки. Поэтому усилия исследователей направлены на разработку требуемых для биомедицинских применений магнитных материалов с высокими магнитными характеристиками, например, с высокой магнитной анизотропией. Так, были синтезированы композиты с использованием гексагонального феррита типа $M$, обладающих высокой магнитной анизотропией, внедренных матрице биологически совместимого гидрокисапатита [8-10], положившие начало новому классу биосовместимых композитов: НАр-гексаферриты.

Среди оксидов железа имеется полиморфная модификация $\varepsilon-\mathrm{Fe}_{2} \mathrm{O}_{3}$ известная с 1934 г., но структура $\varepsilon-\mathrm{Fe}_{2} \mathrm{O}_{3}$ установлена лишь в 1998 г. и уточнена в 2005 г. (см. [11] и ссылки там). До настоящего времени фаза $\varepsilon-\mathrm{Fe}_{2} \mathrm{O}_{3}$ малоизучена, так как отсутствовали способы получения этой фазы в чистом виде, а только в виде включений в другие фазы оксида железа (см. например [12] и ссылки там). Уникальность магнитных свойств фазы $\varepsilon-\mathrm{Fe}_{2} \mathrm{O}_{3}$ оказались в том, что $\varepsilon$ - $\mathrm{Fe}_{2} \mathrm{O}_{3}$ обладает гигантской коэрцитивной силой (до $23 \mathrm{kOe}$ ) при комнатной температуре и высокой магнитной анизотропией [13-16], превышающими все известные значения, что и делает это соединение $\varepsilon$ - $\mathrm{Fe}_{2} \mathrm{O}_{3}$ перспективным для самых разнообразных применений, в том числе и для биомедицины. Однако, получить монофазные частицы $\varepsilon$ - $\mathrm{Fe}_{2} \mathrm{O}_{3}$ очень сложно, так как большинство методов синтеза дает смесь $\varepsilon-\mathrm{Fe}_{2} \mathrm{O}_{3}$ с различным содержанием $\alpha-\mathrm{Fe}_{2} \mathrm{O}_{3}$ и/или $\gamma-\mathrm{Fe}_{2} \mathrm{O}_{3}$. Для синтеза $\varepsilon-\mathrm{Fe}_{2} \mathrm{O}_{3}$, была предложена методика получения наночастиц $\varepsilon$ - $\mathrm{Fe}_{2} \mathrm{O}_{3}$ в матрице мезопористого аморфного кремнезема, обладающего высокопористой структурой, имеющей нанопоры с высокой удельной площадью, 
(см. $[17,18]$ и ссылки там). Такая методика позволяет получать на выходе гораздо больше оксида $\varepsilon-\mathrm{Fe}_{2} \mathrm{O}_{3}$ либо в виде моно фазы (без обнаруженных экспериментально примесей других фаз оксида железа) или с экспериментально обнаруживаемой, но незначительной примесью других полиморфов $\mathrm{Fe}_{2} \mathrm{O}_{3}$ [18]. Пористость матрицы обеспечивает участки образования зародышей наночастиц $\varepsilon$ - $\mathrm{Fe}_{2} \mathrm{O}_{3}$ и изоляция МНЧ друг от друга, предотвращает их агрегацию. Размещение наночастиц $\varepsilon-\mathrm{Fe}_{2} \mathrm{O}_{3}$ в порах матрицы повышает их термическую стабильность. Образование фазы $\varepsilon-\mathrm{Fe}_{2} \mathrm{O}_{3}$ очень чувствительно к условиям синтеза, таким как окислительная способность атмосферы, длительность окисления и/или наличие гидроксильных групп (т.е. избыток воды, высокий коэффициент гидролиза и т.д.) (см. [18] и ссылки там).

Существенным ограничением использования МНЧ в медицине является их биологическая несовместимость с живым организмом. С целью устранения этого препятствия создаются магнитные композиты (МК), в которых МНЧ либо окружены биосовместимым материалом, либо МНЧ внедряются в биосовместимую матрицу. Биосовместимыми веществами являются золото (Au) [19], полимеры, оксид кремния $\left(\mathrm{SiO}_{2}\right)$ [20], оксид титана $\left(\mathrm{TiO}_{2}\right)$ [21] и гидроксиапатит $\left\{\left(\mathrm{Ca}_{10}\left(\mathrm{PO}_{4}\right)_{6}(\mathrm{OH})_{2}\right\}\right.$ [22-24]. Гидроксиапатит (НАр) является биосовместимым потому, что структура НАр аналогична структуре костного материала. Ряд работ посвящен исследованиям свойств композитов НАр-ферриты [10,25-27].

Данная работа посвящена созданию магнитных композитов гидроксиапатит/феррооксиды (HAp/FeOxid) c максимальной долей в ферроксиде фазы $\varepsilon-\mathrm{Fe}_{2} \mathrm{O}_{3}$, обладающей уникальными свойствами. Выбор НАр обусловлен его высокой биологической совместимостью, а также пористостью, что может обеспечить зародышеобразование наночастиц $\varepsilon-\mathrm{Fe}_{2} \mathrm{O}_{3}$. Для изучения свойств МК привлечены различные методы, позволяющие сопоставить информацию о локальных и объемных свойствах МК. Композиты обладают сложной структурой, которую не удается определить, пользуясь каким-либо одним видом анализа, например, рентгенофазовым. Поэтому была использована мессбауэровская спектроскопия, уникальность которой в том, что он является единственным методом, позволяющим провести фазовый анализ ферроооксидов.

\section{2. Методики экспериментов}

Для исследований были синтезированы методом пиролиза ультразвуковой аэрозоли магнитные композитные (MK) частицы HAp/FeOxid, как при различных температурах пиролиза $\left(800,900\right.$ и $\left.1000^{\circ} \mathrm{C}\right)$, так и с разными соотношениями Нар:FeOxid, а именно: 1:1. 1:2 и 1:3. Композиты HAp/FeOxid были получены в два этапа, модификацией приема, описанного в [26]. На первом этапе методом со-осаждения были синтезированы нано- частицы оксидов железа. На втором этапе методом пиролиза ультразвуковой аэрозоли (ПУА) были получены МК НAp/FeOxid. Метод ПУА, это один из эффективных методов синтеза МНЧ и МК, заключающийся в том, что „туман“, полученный с помощью ультразвука из солевого раствора, разлагается в горячей зоне печи с образованием субмикронных частиц сложных оксидов $[26,28]$.

Одной из важнейших проблем при исследованиях оксидов железа является идентификация и разделение фаз оксидов железа: гематита $\left(\alpha-\mathrm{Fe}_{2} \mathrm{O}_{3}\right)$, магнетита $\left(\mathrm{Fe}_{3} \mathrm{O}_{4}\right)$, маггемита $\left(\gamma-\mathrm{Fe}_{2} \mathrm{O}_{3}\right)$, вюстита $(\mathrm{FeO})$, гетита $(\alpha-\mathrm{FeOOH})$, акаганита $(\beta$-FeOOH). Вюстит имеет кубическую кристаллическую структуру, гематит - структуру типа корунда. Перечисленные оксиды железа практически различимы рентгеновским методом. Уникальная чувствительность мессбауэровской спектроскопии позволяет изучать изменения локального окружения атомов Fe в кристаллической решетке. Получаемые из положений спектральных линий в МС параметры сверхтонких взаимодействий, такие как изомерный химический сдвиг (IS), квадрупольное расщепление (QS), квадрупольный сдвиг $(\varepsilon \mathrm{Q})$ и эффективное магнитное поле $(\mathrm{Bhf})$, дают важную информацию об электронной плотности, ее симметрии и магнитных свойствах материала. Метод также позволяет извлечь ценные характеристики материала из ширин спектральных линий, их относительной интенсивности, асимметрии спектра и температурной и полевой зависимости сверхтонких параметров. Из мессбауэровских спектров извлекается информация о валентности и спиновых состояниях железа, о количестве неэквивалентных положений ионов $\mathrm{Fe}$ в кристаллической решетке, о координации $\mathrm{Fe}$ в кристаллографических положениях, стехиометрии, ориентации магнитных моментов, магнитной анизотропии, о степени, типе и температуре магнитного упорядочения [29].

В случае полиморфных оксидов железа (III) мссбауэровская спектроскопия ${ }^{57} \mathrm{Fe}$ обеспечивает четкое разделение отдельных спектральных компонентов, принадлежащих $\alpha-\mathrm{Fe}_{2} \mathrm{O}_{3}, \beta-\mathrm{Fe}_{2} \mathrm{O}_{3}, \gamma-\mathrm{Fe}_{2} \mathrm{O}_{3}$ и $\varepsilon-\mathrm{Fe}_{2} \mathrm{O}_{3}$, из-за различной кристаллической структуры и магнитного поведения этих фаз, отраженных в их характерных мёссбауэровских сверхтонких параметрах (см. например $[18,30])$. Таким образом, мессбауэровская спектроскопия является единственным методом, позволяющим различить фазы различных ферроксидов в образце и, таким образом, решить важную проблему фазового анализа оксидов железа.

Исследования синтезированных МК HAp/FeOxid были проведены с помощью Мессбауэровской спектроскопии c регистрацией гамма-излучения, поглощаемого изотопом ${ }^{57} \mathrm{Fe}$, в геометрии пропускания через исследуемый образец. Опорный сигнал в системе движения доплеровского модулятора в спектрометре имел форму треугольника для задания скорости с постоянным ускорением. Мессбауэровским источником гамма-излучения служил $\mathrm{Co}^{57}$ активностью $20 \mathrm{mCi}$ в матрице родия ( $\left.\mathrm{Rd}\right)$. 
Таблица 1. Рассчитанные из мессбауэровских спектров комнатной температуры магнитных композитов НАр/FеОхіd концентрации $1 / 1$, синтезированных при разных температурах, величины эффективных магнитных полей $\left(H_{\text {eff }}\right)$, изомерных химических сдвигов (IS) относительно $\alpha$-Fe, квадрупольных расщеплений $(\mathrm{QS})$, ширин $(\Gamma)$ и площадей $(A)$ линий поглощения

\begin{tabular}{|c|c|c|c|c|c|}
\hline$T_{\mathrm{ann}}=1000^{\circ} \mathrm{C}$ & $\Gamma, \mathrm{mm} / \mathrm{s}$ & IS, $\mathrm{mm} / \mathrm{s}$ & $\mathrm{QS}, \mathrm{mm} / \mathrm{s}$ & $H_{\text {eff }}, \mathrm{kOe}$ & $A, \%$ \\
\hline $\begin{array}{c}\beta \text {-FеООН } \\
\gamma \text {-Fe } \mathrm{O}_{3} \\
\mathrm{Fe}_{3} \mathrm{O}_{4} A \text {-подр } \\
\mathrm{Fe}_{3} \mathrm{O}_{4} B \text {-подр }+\varepsilon-\mathrm{Fe}_{2} \mathrm{O}_{3} A \text {-подр } \\
\varepsilon-\mathrm{Fe}_{2} \mathrm{O}_{3} B \text {-подр } \\
\varepsilon-\mathrm{Fe}_{2} \mathrm{O}_{3} C \text {-подр } \\
\varepsilon-\mathrm{Fe}_{2} \mathrm{O}_{3} D \text {-подр }\end{array}$ & $\begin{array}{l}0.668 \pm 0.000 \\
0.697 \pm 0.049 \\
0.492 \pm 0.033 \\
0.481 \pm 0.025 \\
0.480 \pm 0.033 \\
0.518 \pm 0.035 \\
0.474 \pm 0.059 \\
0.475 \pm 0.035\end{array}$ & $\begin{array}{l}0.317 \pm 0.057 \\
0.226 \pm 0.033 \\
0.279 \pm 0.050 \\
0.255 \pm 0.015 \\
0.648 \pm 0.037 \\
0.596 \pm 0.020 \\
0.481 \pm 0.047 \\
0.470 \pm 0.440\end{array}$ & $\begin{array}{l}0.896 \pm 0.095 \\
1.225 \pm 0.084 \\
0.068 \pm 0.080 \\
0.065 \pm 0.032 \\
0.068 \pm 0.052 \\
0.030 \pm 0.039 \\
0.002 \pm 0.093 \\
0.113 \pm 0.875\end{array}$ & $\begin{array}{c}- \\
- \\
487 \pm 1.0 \\
474 \pm 1.0 \\
455 \pm 1.0 \\
411 \pm 1.0 \\
382 \pm 1.0 \\
283 \pm 1.0\end{array}$ & $\begin{array}{r}14 \\
27 \\
9 \\
18 \\
14 \\
11 \\
4 \\
4\end{array}$ \\
\hline$T_{\mathrm{ann}}=900^{\circ} \mathrm{C}$ & $\Gamma, \mathrm{mm} / \mathrm{s}$ & $\mathrm{IS}, \mathrm{mm} / \mathrm{s}$ & $\mathrm{QS}, \mathrm{mm} / \mathrm{s}$ & $H_{\text {eff }}, \mathrm{kOe}$ & $A, \%$ \\
\hline $\begin{array}{c}\beta \text {-FеООН } \\
\gamma \text { - } \mathrm{Fe}_{2} \mathrm{O}_{3} \\
\mathrm{Fe}_{3} \mathrm{O}_{4} A \text {-подр } \\
\mathrm{Fe}_{3} \mathrm{O}_{4} B \text {-подр }+\varepsilon \text { - } \mathrm{Fe}_{2} \mathrm{O}_{3} A \text {-подр } \\
\varepsilon-\mathrm{Fe}_{2} \mathrm{O}_{3} B \text {-подр } \\
\varepsilon-\mathrm{Fe}_{2} \mathrm{O}_{3} C \text {-подр } \\
\varepsilon-\mathrm{Fe}_{2} \mathrm{O}_{3} D \text {-подр }\end{array}$ & $\begin{array}{l}0.568 \pm 0.000 \\
0.588 \pm 0.000 \\
0.499 \pm 0.000 \\
0.474 \pm 0.000 \\
0.489 \pm 0.000 \\
0.493 \pm 0.000 \\
0.504 \pm 0.000 \\
0.483 \pm 0.000\end{array}$ & $\begin{array}{l}0.385 \pm 0.000 \\
0.284 \pm 0.011 \\
0.367 \pm 0.008 \\
0.091 \pm 0.024 \\
0.746 \pm 0.013 \\
0.461 \pm 0.017 \\
0.384 \pm 0.014 \\
0.402 \pm 0.018\end{array}$ & $\begin{array}{l}0.490 \pm 0.000 \\
0.900 \pm 0.016 \\
0.023 \pm 0.016 \\
0.401 \pm 0.050 \\
0.487 \pm 0.026 \\
0.352 \pm 0.034 \\
0.099 \pm 0.027 \\
0.211 \pm 0.035\end{array}$ & $\begin{array}{c}- \\
- \\
489 \pm 1.0 \\
470 \pm 1.0 \\
448 \pm 1.0 \\
421 \pm 1.0 \\
346 \pm 1.0 \\
259 \pm 1.0\end{array}$ & $\begin{array}{r}13 \\
16 \\
25 \\
8 \\
10 \\
9 \\
11 \\
9\end{array}$ \\
\hline$T_{\mathrm{ann}}=800^{\circ} \mathrm{C}$ & $\Gamma, \mathrm{mm} / \mathrm{s}$ & $\mathrm{IS}, \mathrm{mm} / \mathrm{s}$ & $\mathrm{QS}, \mathrm{mm} / \mathrm{s}$ & $H_{\text {eff }}, \mathrm{kOe}$ & $A, \%$ \\
\hline $\begin{array}{c}\beta \text {-FеООН } \\
\gamma \text { - } \mathrm{Fe}_{2} \mathrm{O}_{3} \\
\mathrm{Fe}_{3} \mathrm{O}_{4} A \text {-подр } \\
\mathrm{Fe}_{3} \mathrm{O}_{4} B \text {-подр }+\varepsilon-\mathrm{Fe}_{2} \mathrm{O}_{3} A \text {-подр } \\
\varepsilon-\mathrm{Fe}_{2} \mathrm{O}_{3} B \text {-подр } \\
\mathcal{\varepsilon}-\mathrm{Fe}_{2} \mathrm{O}_{3} C \text {-подр } \\
\varepsilon-\mathrm{Fe}_{2} \mathrm{O}_{3} D \text {-подр }\end{array}$ & $\begin{array}{l}0.479 \pm 0.000 \\
0.507 \pm 0.033 \\
0.492 \pm 0.029 \\
0.481 \pm 0.032 \\
0.480 \pm 0.035 \\
0.518 \pm 0.037 \\
0.474 \pm 0.259 \\
0.475 \pm 0.039\end{array}$ & $\begin{array}{l}0.400 \pm 0.010 \\
0.079 \pm 0.014 \\
0.371 \pm 0.016 \\
0.300 \pm 0.010 \\
0.729 \pm 0.013 \\
0.565 \pm 0.011 \\
0.472 \pm 0.018 \\
0.541 \pm 0.047\end{array}$ & $\begin{array}{l}0.447 \pm 0.014 \\
1.515 \pm 0.032 \\
0.203 \pm 0.034 \\
0.042 \pm 0.020 \\
0.408 \pm 0.026 \\
0.096 \pm 0.022 \\
0.068 \pm 0.032 \\
0.123 \pm 0.096\end{array}$ & $\begin{array}{c}- \\
- \\
491 \pm 1.0 \\
478 \pm 1.0 \\
451 \pm 1.0 \\
411 \pm 1.0 \\
362 \pm 1.0 \\
261 \pm 1.0\end{array}$ & $\begin{array}{r}14 \\
20 \\
10 \\
18 \\
10 \\
16 \\
8 \\
4\end{array}$ \\
\hline
\end{tabular}

Скоростная шкала калибровалась с использованием при комнатной температуре фольги $\alpha$-Fе толщиной $6 \mu \mathrm{m}$, а для более высокой точности калибровка проводилась с помощью лазерного интерферометра. МС исследуемых МК HAp/FeOхіd были получены при комнатной температуре. Математическая обработка экспериментальных МС проводилась с использованием программы [31]. Расхождение теоретических значений параметров сверхтонких взаимодействий определяются из статистических отклонений, предоставленных программой математической обработки [31]. Микроструктура МК изучалась с помощью сканирующего электронного микроскопа (СЭМ) (JSM-5510SV; JEOL) и просвечивающего сканирующего электронного микроскопа (ПСЭМ) (JEM-2100F; JEOL).

\section{3. Результаты и обсуждение}

Экспериментальные мессбауэровские спектры (MC) магнитных композитов HAp/FeOxid взятых в соотношении компонентов $1: 1$ и отожженных при температурах 800,900 и $1000^{\circ} \mathrm{C}$, показаны на рис. 1 . На рис. 2 приведены MC комнатной температуры МКЧ HAp/FeOxid подготовленных в соотношениях $1: 3,1: 2$ и $1: 1$ и отожженных при температуре $1000^{\circ} \mathrm{C}$.

Как видно на рис. 1 и 2, MC MК HAp/FeOxid состоят из линий зеемановских секстиплетов и наложенных на них в области нуля скоростей интенсивных дублетов. При температуре отжига МК при $800^{\circ} \mathrm{C}$ (рис. 1) отдельные компоненты зеемановских секстиплетов МС плохо разрешаются. Ширины линий МС указывают на то, что частицы в образце распределены по размерам. При повышении температуры отжига МК линии секстиплетов становятся более четкими, что связанно с лучшей кристаллизацией оксидов. При температуре отжига $1000^{\circ} \mathrm{C}$ линии МC MК HAp/FeOxid становятся достаточно хорошо разрешимы, что позволяет провести фазовый анализ синтезированных МК на более качественном уровне.

Математическая обработка экспериментальных МС (рис. 1 и 2) проведена с помощью программы [31]. Для максимальной достоверности обработки МС использовались несколько различных возможных моделей. Наилучшее соответствие с экспериментальными спектрами было получено при использовании модельных спектров с лоренцевой формой линий, состоящих из 
Таблица 2. Рассчитанные из мессбауэровских спектров комнатной температуры магнитных композитов НАр/FеОхіd, синтезированных при $1000^{\circ} \mathrm{C}$ при разных концентрациях феррооксида величины эффективных магнитных полей ( $\left.H_{\text {eff }}\right)$, изомерных химических сдвигов (IS) относительно $\alpha$-Fe, квадрупольных расщеплений $(\mathrm{QS})$, ширин $(\Gamma)$ и площадей $(A)$ линий поглощения

\begin{tabular}{|c|c|c|c|c|c|}
\hline HAp: $\mathrm{Fe}_{x} \mathrm{O}_{y}=1: 1$ & $\Gamma, \mathrm{mm} / \mathrm{s}$ & IS, $\mathrm{mm} / \mathrm{s}$ & $\mathrm{QS}, \mathrm{mm} / \mathrm{s}$ & $H_{\text {eff }} \mathrm{kOe}$ & A, $\%$ \\
\hline $\begin{array}{c}\beta \text {-FeOОH }+ \\
\gamma \text { - } \mathrm{Fe}_{2} \mathrm{O}_{3} \mathrm{spm} \\
\gamma \text { - } \mathrm{Fe}_{2} \mathrm{O}_{3} \\
\mathrm{Fe}_{3} \mathrm{O}_{4} A \text {-подр } \\
\mathrm{Fe}_{3} \mathrm{O}_{4} B \text {-подр }+\varepsilon \text { - } \mathrm{Fe}_{2} \mathrm{O}_{3} A \text {-подр } \\
\varepsilon-\mathrm{Fe}_{2} \mathrm{O}_{3} B \text {-подр } \\
\varepsilon-\mathrm{Fe}_{2} \mathrm{O}_{3} C \text {-подр } \\
\varepsilon-\mathrm{Fe}_{2} \mathrm{O}_{3} D \text {-подр }\end{array}$ & $\begin{array}{l}0.668 \pm 0.000 \\
0.697 \pm 0.049 \\
0.492 \pm 0.033 \\
0.481 \pm 0.025 \\
0.480 \pm 0.033 \\
0.518 \pm 0.035 \\
0.474 \pm 0.059 \\
0.475 \pm 0.035\end{array}$ & $\begin{array}{l}0.317 \pm 0.057 \\
0.226 \pm 0.033 \\
0.279 \pm 0.050 \\
0.255 \pm 0.015 \\
0.648 \pm 0.037 \\
0.596 \pm 0.020 \\
0.481 \pm 0.047 \\
0.470 \pm 0.440\end{array}$ & $\begin{array}{l}0.896 \pm 0.095 \\
1.225 \pm 0.084 \\
0.068 \pm 0.080 \\
0.065 \pm 0.032 \\
0.068 \pm 0.052 \\
0.030 \pm 0.039 \\
0.002 \pm 0.093 \\
0.113 \pm 0.875\end{array}$ & $\begin{array}{c}- \\
- \\
487 \pm 1.0 \\
474 \pm 1.0 \\
451 \pm 1.0 \\
418 \pm 1.0 \\
372 \pm 1.0 \\
273 \pm 1.0\end{array}$ & $\begin{array}{r}14 \\
27 \\
9 \\
18 \\
14 \\
11 \\
4 \\
4\end{array}$ \\
\hline HAp : $\mathrm{Fe}_{x} \mathrm{O}_{y}=1: 2$ & $\Gamma, \mathrm{mm} / \mathrm{s}$ & $\mathrm{IS}, \mathrm{mm} / \mathrm{s}$ & $\mathrm{QS}, \mathrm{mm} / \mathrm{s}$ & $H_{\text {eff }} \mathrm{kOe}$ & $\mathrm{A}, \%$ \\
\hline $\begin{array}{c}\beta \text {-FeOОH }+ \\
\gamma \text { - } \mathrm{Fe}_{2} \mathrm{O}_{3} \text { spm } \\
\gamma \text { - } \mathrm{Fe}_{2} \mathrm{O}_{3} \\
\mathrm{Fe}_{3} \mathrm{O}_{4} A \text {-подр } \\
\mathrm{Fe}_{3} \mathrm{O}_{4} B \text {-подр }+\varepsilon \text { - } \mathrm{Fe}_{2} \mathrm{O}_{3} A \text {-подр } \\
\varepsilon-\mathrm{Fe}_{2} \mathrm{O}_{3} B \text {-подр } \\
\varepsilon-\mathrm{Fe}_{2} \mathrm{O}_{3} C \text {-подр } \\
\varepsilon-\mathrm{Fe}_{2} \mathrm{O}_{3} D \text {-подр }\end{array}$ & $\begin{array}{l}0.479 \pm 0.000 \\
0.507 \pm 0.048 \\
0.492 \pm 0.036 \\
0.381 \pm 0.033 \\
0.480 \pm 0.033 \\
0.518 \pm 0.034 \\
0.474 \pm 0.059 \\
0.475 \pm 0.055\end{array}$ & $\begin{array}{l}0.342 \pm 0.007 \\
0.009 \pm 0.026 \\
0.363 \pm 0.016 \\
0.269 \pm 0.009 \\
0.546 \pm 0.016 \\
0.493 \pm 0.015 \\
0.511 \pm 0.015 \\
0.527 \pm 0.045\end{array}$ & $\begin{array}{l}0.572 \pm 0.012 \\
1.656 \pm 0.037 \\
0.221 \pm 0.032 \\
0.065 \pm 0.019 \\
0.081 \pm 0.032 \\
0.100 \pm 0.029 \\
0.075 \pm 0.030 \\
0.276 \pm 0.087\end{array}$ & $\begin{array}{c}- \\
- \\
503 \pm 1.0 \\
476 \pm 1.0 \\
447 \pm 1.0 \\
412 \pm 1.0 \\
371 \pm 1.0 \\
266 \pm 1.0\end{array}$ & $\begin{array}{r}14 \\
6 \\
16 \\
18 \\
12 \\
11 \\
8 \\
15\end{array}$ \\
\hline HAp : $\mathrm{Fe}_{x} \mathrm{O}_{y}=1: 3$ & $\Gamma, \mathrm{mm} / \mathrm{s}$ & IS, $\mathrm{mm} / \mathrm{s}$ & $\mathrm{QS}, \mathrm{mm} / \mathrm{s}$ & $H_{\text {eff }} \mathrm{kOe}$ & A, $\%$ \\
\hline $\begin{array}{c}\beta \text {-FeOОH }+ \\
\gamma \text { - } \mathrm{Fe}_{2} \mathrm{O}_{3} \text { spm } \\
\gamma \text { - } \mathrm{Fe}_{2} \mathrm{O}_{3} \\
\mathrm{Fe}_{3} \mathrm{O}_{4} A \text {-подр } \\
\mathrm{Fe}_{3} \mathrm{O}_{4} B \text {-подр }+\varepsilon \text { - } \mathrm{Fe}_{2} \mathrm{O}_{3} A \text {-подр } \\
\varepsilon-\mathrm{Fe}_{2} \mathrm{O}_{3} B \text {-подр } \\
\varepsilon-\mathrm{Fe}_{2} \mathrm{O}_{3} c \text {-подр } \\
\varepsilon-\mathrm{Fe}_{2} \mathrm{O}_{3} D \text {-подр }\end{array}$ & $\begin{array}{l}0.479 \pm 0.032 \\
0.507 \pm 0.034 \\
0.492 \pm 0.031 \\
0.381 \pm 0.029 \\
0.480 \pm 0.033 \\
0.518 \pm 0.036 \\
0.524 \pm 0.040 \\
0.725 \pm 0.051\end{array}$ & $\begin{array}{l}0.335 \pm 0.004 \\
0.015 \pm 0.018 \\
0.380 \pm 0.007 \\
0.273 \pm 0.004 \\
0.568 \pm 0.006 \\
0.524 \pm 0.009 \\
0.502 \pm 0.010 \\
0.397 \pm 0.036\end{array}$ & $\begin{array}{l}0.572 \pm 0.006 \\
1.626 \pm 0.027 \\
0.235 \pm 0.015 \\
0.077 \pm 0.009 \\
0.088 \pm 0.012 \\
0.005 \pm 0.018 \\
0.041 \pm 0.019 \\
0.091 \pm 0.072\end{array}$ & $\begin{array}{c}- \\
- \\
504 \pm 1.0 \\
477 \pm 1.0 \\
448 \pm 1.0 \\
394 \pm 1.0 \\
350 \pm 1.0 \\
262 \pm 1.0\end{array}$ & $\begin{array}{r}14 \\
5 \\
14 \\
19 \\
17 \\
10 \\
7 \\
15\end{array}$ \\
\hline
\end{tabular}

пяти зеемановских секстиплетов и двух дублетов. Параметры сверхтонких взаимодействий (СТВ), полученные при обработке экспериментальных МС исследуемых МК приведены в табл. 1 и 2, где они обозначены следующим образом: IS - изомерный химический сдвиг, рассчитаный по отношению к $\alpha$-Fe $(\mathrm{mm} / \mathrm{s}), \mathrm{QS}$ - квадрупольное расщепление $(\mathrm{mm} / \mathrm{s}), H_{\text {eff }}$ - эффективное магнитное поле $(\mathrm{kOe}), \Gamma(\mathrm{mm} / \mathrm{s})$ - ширины линий, \% - процентное содержание компоненты. Относительные количества фаз, приведенные в табл. 1 и 2, получены из МС при допущении, что коэффициент безотдаточного поглощения одинаков для всех ионов железа, находящихся в различных подрешетках.

Анализ полученных параметров СТВ, представленных в табл. 1 и 2, показал, что секстиплет с наибольшей величиной эффективного магнитного поля (обозначенный на рис. 1 и 2 цифрой 1) соответствует фазе маггемита $\left(\gamma-\mathrm{Fe}_{2} \mathrm{O}_{3}\right)$ (см. [30] и ссылки там). Секстиплеты со значениями эффективных магнитных полей, равными $\sim 483$ и $\sim 446 \mathrm{kOe}$, ближе к величинам для макроскопических кристаллов магнетита $\left(\mathrm{Fe}_{3} \mathrm{O}_{4}\right)(\sim 492$ и $~ 461 \mathrm{kOe})$. Секстиплет с более высоким магнитным полем относится (обозначенный на рис. 1 и 2 цифрой 2) к октаэдрическим положениям ионов железа $A$-подрешетки в магнетите за счет роста дипольных полей, возникающих в результате отклонения от кубической симметрии и ковалентной природы тетраэдрических связей. Зеемановский секстиплет (обозначенный на рис. 1 и рис. 2 цифрой 3) относятся к ионам $\mathrm{Fe}$ в тетраэдрической $B$-подрешетке магнетита. Величины эффективных полей, приведенные для магнетита в табл. 1 и 2, немного меньше значений для макрокристаллов потому, что исследуемые МК HAp/FeOxid являются мелкими частицами. Это подтверждается совпадением параметров СТВ для данных секстиплетов с величинами, приведенными для $\mathrm{Fe}_{3} \mathrm{O}_{4}$ в литературе (см., например, [30]] и ссылки там).

Зеемановские секстиплеты с меньшими величинами эффективных магнитных полей $(\sim 455, \sim 425, \sim 480$ и $\sim 280 \mathrm{kOe})$, относятся к ионам железа в трех тетраэдрических и одной октаэдрической подрешетках фазы $\varepsilon-\mathrm{Fe}_{2} \mathrm{O}_{3}$, соответственно. Эти секстиплеты обозначены на рис. 1 и 2 цифрами $3,4,5$ и 6. Такое описание спектров согласуется с кристаллической структурой $\varepsilon-\mathrm{Fe}_{2} \mathrm{O}_{3}$, в которой ионы железа распределены по четырем неэквивалентным положениям, каждое из которых формирует секстиплет [18,30,32,33]. Следует отметить, что линии секстиплета с максимальной величиной эффективного поля $\left(H_{\text {eff }}\right)$, принадлежащие фазе $\varepsilon-\mathrm{Fe}_{2} \mathrm{O}_{3}$, совпадают 


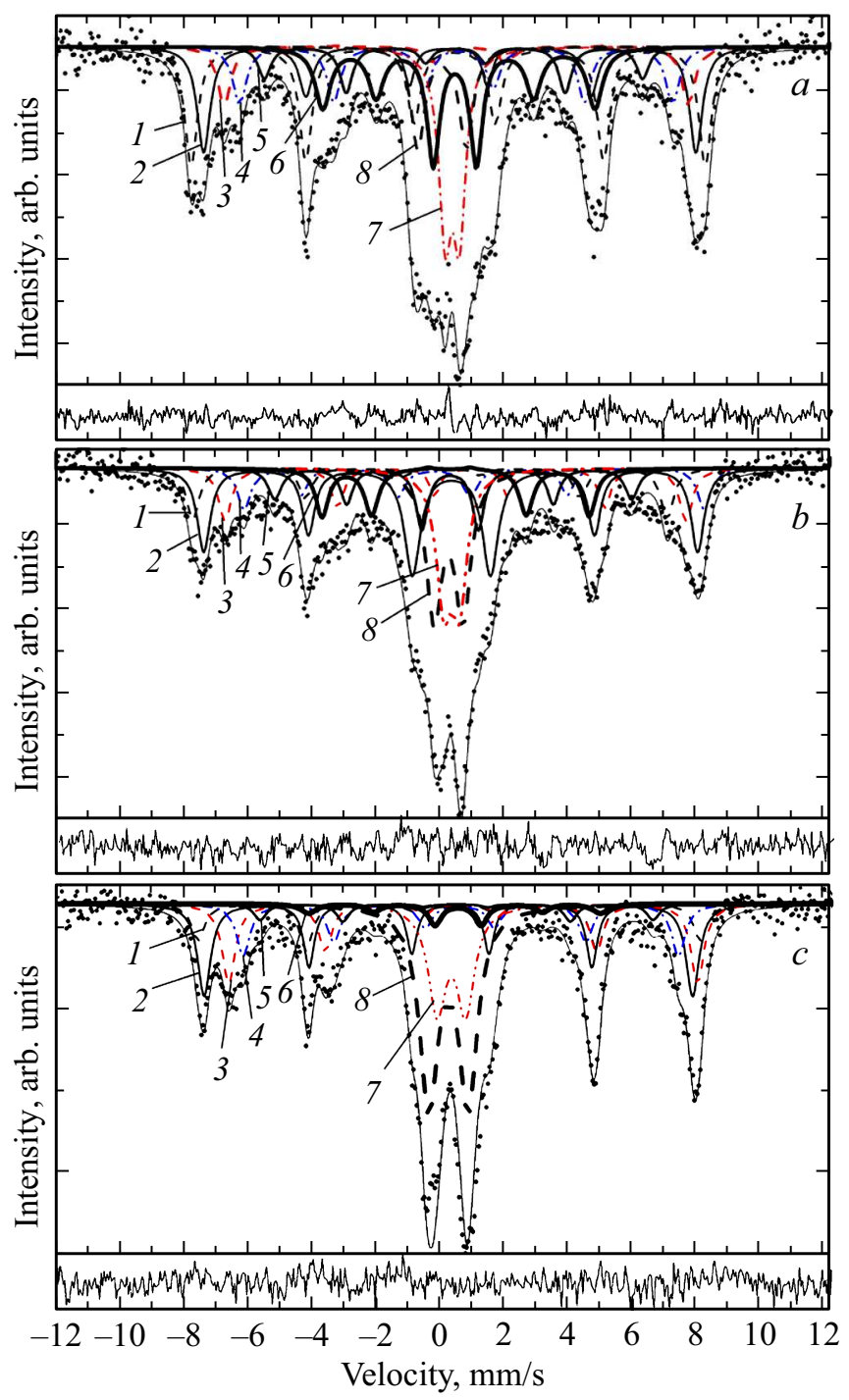

Рис. 1. Мессбауэровские спектры композитов гидроксиапатитферрооксиды синтезированных в соотношении $1: 1$ при температурах отжига: $800^{\circ} \mathrm{C}-a, 900^{\circ} \mathrm{C}-b$ и $1000^{\circ} \mathrm{C}-c$. Точками показаны экспериментальные значения, тонкой сплошной линией - модельная кривая. Линии зеемановского расщепления, обозначенные цифрой 1 относятся к фазе маггемита $\left(\gamma-\mathrm{Fe}_{2} \mathrm{O}_{3}\right)$, 2 - к тетраэдрическим позициям ионов $\mathrm{Fe}$ в структуре магнетита $\left(\mathrm{Fe}_{3} \mathrm{O}_{4}\right), 3-$ к октаэдрическим положениям ионов $\mathrm{Fe}$ в структуре магнетита $\left(\mathrm{Fe}_{3} \mathrm{O}_{4}\right)$, линии $3,4,5$ и $6-$ к положениям ионов $\mathrm{Fe}$ в структуре $\mathcal{\varepsilon}-\mathrm{Fe}_{2} \mathrm{O}_{3}$. Линии парамагнитной фазы $\beta$-FeOОН обозначены 7 и 8 . Под каждым спектром показаны разности между экспериментальными данными и теоретической моделью.

по положениям с линиями секстиплета $B$-подрешетки магнетита $\left(\mathrm{Fe}_{3} \mathrm{O}_{4}\right)$ и они обозначены цифрой 3 на рис. 1 и 2. Полученные мессбауэровские спектры фазы $\varepsilon$ - $\mathrm{Fe}_{2} \mathrm{O}_{3}$ (рис. 1 и 2) при комнатной температуре описываются четырьмя секстетами с отношением спектральных линий в секстиплетах, как $2: 1: 1$, что согласуется с данными работ [18,30,32,33]. В соответствии со значениями изомерных сдвигов и квадрупольных расщеплений па-

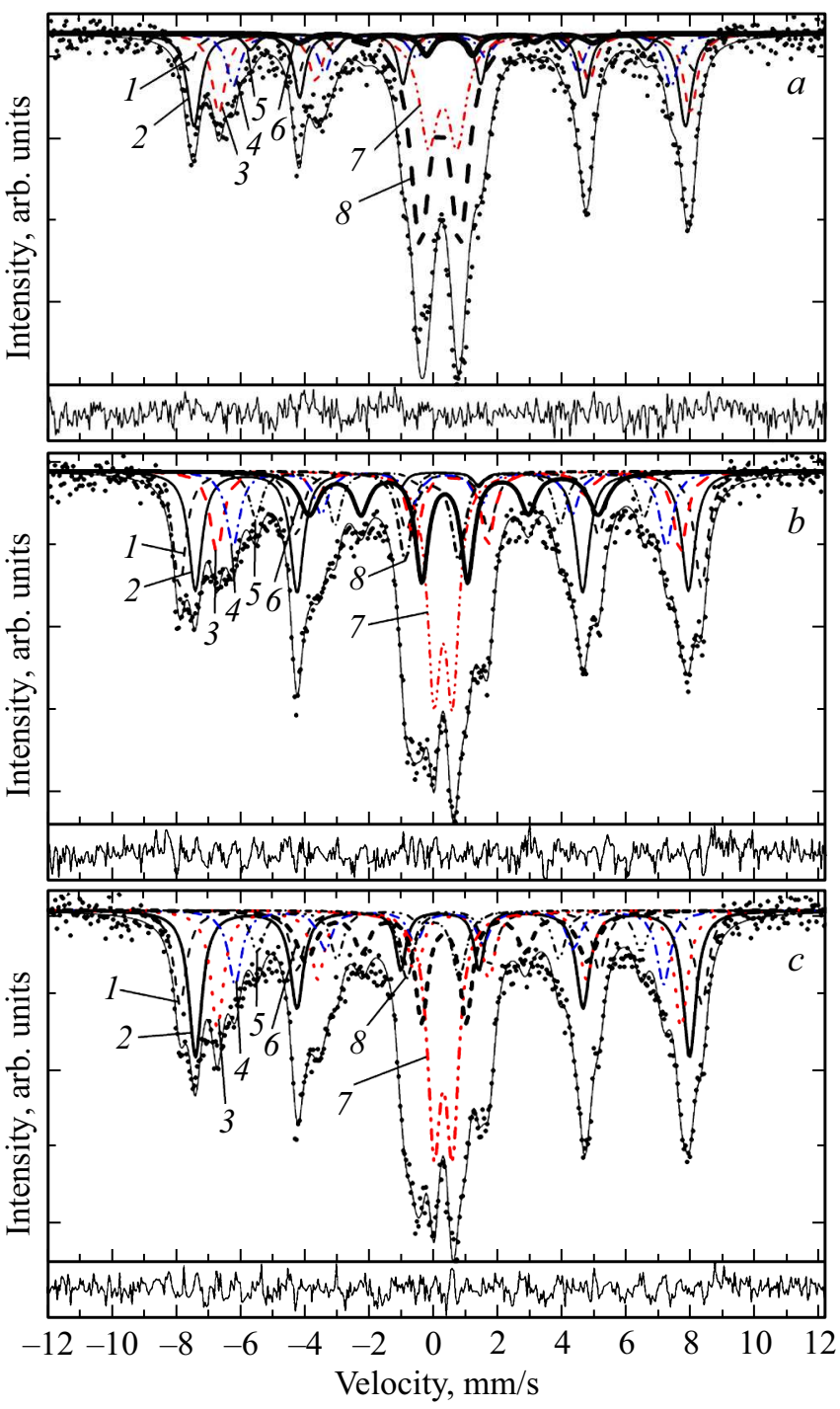

Рис. 2. Мессбауэровские спектры отожженных при температуре $1000^{\circ} \mathrm{C}$ композитов гидроксиапатит-ферроокиды, взятых в соотношениях $1: 1-a, 1: 2-b$ и $1: 3-c$. Точками показаны экспериментальные значения, тонкой сплошной линией - модельная кривая. Линии зеемановского расщепления, обозначенные цифрой 1 относятся к фазе маггемита $\left(\gamma-\mathrm{Fe}_{2} \mathrm{O}_{3}\right)$, 2 - к тетраэдрическим позициям ионов $\mathrm{Fe}$ в структуре магнетита $\left(\mathrm{Fe}_{3} \mathrm{O}_{4}\right), 3-$ к октаэдрическим положениям ионов $\mathrm{Fe}$ в структуре магнетита $\left(\mathrm{Fe}_{3} \mathrm{O}_{4}\right)$, линии $3,4,5$ и 6 - к положениям ионов $\mathrm{Fe}$ в структуре $\varepsilon-\mathrm{Fe}_{2} \mathrm{O}_{3}$. Линии парамагнитной фазы $\beta$ - $\mathrm{FeOOH}$ обозначены 7 и 8 . Под каждым спектром показаны разности между экспериментальными данными и теоретической моделью.

рамагнитные дублеты в области нуля скоростей были приписаны фазе $\beta$ - $\mathrm{FeOOH}$, находящейся при комнатной температуре в парамагнитной фазе (см. [34] и ссылки там). Каждый дублет представляет собой парамагнитную составляющую для $\mathrm{Fe}^{3+}$. Параметры СТВ дублета с меньшей величиной квадрупольного расщепления, обозначенные цифрой 7 , совпадают со значениями СТВ для наночастиц маггемита в парамагнитном состоянии. 
Значения изомерных сдвигов (IS) (см. табл. 1 и 2) находятся в диапазоне от 0.19 до $0.26 \mathrm{~mm} / \mathrm{s}$, а значения квадрупольного расщепления (QS) в диапазоне от -0.09 до $0.74 \mathrm{~mm} / \mathrm{s}$, указывая, что ионы $\mathrm{Fe}^{3+}$ находятся в высокоспиновом состоянии. Ионы $\mathrm{Fe}^{2+}$ имеют гораздо большее значение химического сдвига (от 0.9 до $1.1 \mathrm{~mm} / \mathrm{s}$ ). На экспериментальных МС линий с такими величинами IS обнаружено не было, следовательно в исследуемых MK HAp/FeOxid ионы $\mathrm{Fe}^{2+}$ отсутствуют.

При увеличении концентрации ферроооксидов в МК HAp/FeOxid, как видно на рис. 2, интенсивности линии зеемановских секстиплетов, по сравнению с интенсивностями линий в области нуля скоростей, повышаются и становятся лучше разрешимыми. С повышением температуры отжига также существенно меняется форма линии в области нуля скоростей. Так, интенсивность линий дублета, обозначенного цифрой 7, существенно понижается как при повышении температуры отжига MК HAp/FeOxid (рис. 1), так и при понижении количества феррооксидов в композите. Это указывает на увеличение количества парамагнитной фазы маггемита $\left(\gamma-\mathrm{Fe}_{2} \mathrm{O}_{3}\right)$ и акаганита $(\beta-\mathrm{FeOOH})$ в МК при повышении концентрации феррооксидов. Повышение температуры отжига МК приводит к преобразованию парамагнитной фазы маггемита в магнииоупорядоченную фазу феррооксидов.

Таким образом, оксид железа в синтезированных МК состоит из фаз, принадлежащих феррооксидам: $\gamma$ - $\mathrm{Fe}_{2} \mathrm{O}_{3}$, $\mathrm{Fe}_{3} \mathrm{O}_{4}, \varepsilon-\mathrm{Fe}_{2} \mathrm{O}_{3}$ и $\beta$-FеООН. Следует отметить, что результаты фазового анализа на основании данных месбауэровской спектроскопии не согласуются с данными, полученными на основании рентгенограмм синтезированных МК HAp/FeOxid [26,27].

Оксиды железа это $\mathrm{Fe}_{3} \mathrm{O}_{4}$ и $\mathrm{Fe}_{2} \mathrm{O}_{3}$, существующие в четырех различных кристаллических структурах: $\gamma$-, $\varepsilon$-, $\beta-, \alpha-\mathrm{Fe}_{2} \mathrm{O}_{3}[24,32,35]$. Фазы $\mathrm{Fe}_{3} \mathrm{O}_{4}, \gamma-\mathrm{Fe}_{2} \mathrm{O}_{3}$ и $\alpha-\mathrm{Fe}_{2} \mathrm{O}_{3}$ хорошо изучены и широко применяются в промышленности $[3,36,37]$. Оксид $\varepsilon-\mathrm{Fe}_{2} \mathrm{O}_{3}$ в чистом виде редко встречаются в природе. Уникальность фазы $\varepsilon-\mathrm{Fe}_{2} \mathrm{O}_{3}$ в очень большой величине коэрцитивного поля [13-16], что привлекает большое внимание из-за возможностей разнообразных применений этого соединения. С кристаллографической точки зрения $\varepsilon-\mathrm{Fe}_{2} \mathrm{O}_{3}$ имеет орторомбическую кристаллическую структуру с пространственной группой Pna21 и проявляет ферримагнитную особенность ниже $T_{C} \approx 495 \mathrm{~K}$ [18]. Кристаллическая структура $\varepsilon-\mathrm{Fe}_{2} \mathrm{O}_{3}$ содержит четыре кристаллографически неэквивалентных положения ионов железа, из которых три разных октаэдрических (обозначаемых как $A$-, $B$ - и $C$-подрешетки) и одно тетраэдрическое ( $D$-подрешетка). MC-фазы $\varepsilon-\mathrm{Fe}_{2} \mathrm{O}_{3}$ при комнатной температуре состоит из четырех зеемановских секстиплетов [19,37-39]. Спектральные линии, принадлежащие ионам $\mathrm{Fe}$, занимающим тетраэдрические и октаэдрические подрешетки при $300 \mathrm{~K}$ на $\mathrm{MC} \varepsilon-\mathrm{Fe}_{2} \mathrm{O}_{3}$ четко различаются, главным образом из-за существенно меньшей величины эффективного магнитного поля на ионах железа в тетраэдрических положениях [19,37,38]. Относительные интенсивности линий в отдельном секстиплете составляют приблизительно $3: 2: 1: 1: 1: 2: 3$, что соответствует результатам для порошкообразных образцов со случайным распределением ориентации магнитных моментов, поскольку все возможные ориентации моментов равновероятны, в пределах всего $4 \pi$ телесного угла.

Значения изомерных сдвигов (IS) (табл. 1 и 2) для всех четырех секстиплетов в $\mathrm{MC} \varepsilon-\mathrm{Fe}_{2} \mathrm{O}_{3}$ находятся в диапазоне, соответствующем ионам $\mathrm{Fe}^{3+}$ в высокоспиновом состоянии. Меньшее значение IS для секстета $D$-подрешетки, по сравнению со значениями IS для других трех компонентов с магнитным расщеплением линий, указывает на тетраэдрическую координацию атомов $\mathrm{Fe}$ в структуре $\varepsilon-\mathrm{Fe}_{2} \mathrm{O}_{3}$. Разница между значениями IS для октаэдрических и тетраэдрических ионов $\mathrm{Fe}$ в $\varepsilon-\mathrm{Fe}_{2} \mathrm{O}_{3}$ составляет $\sim 0.12 \mathrm{~mm} / \mathrm{s}$, что полностью согласуется с полученной разницей между величинами IS для тетраэдрических и октаэдрических ионов железа в $\gamma-\mathrm{Fe}_{2} \mathrm{O}_{3}$ (см. например [38]). Незначительность величины квадрупольных расщеплений (QS) для $A$ и $B$ ионов железа в $\varepsilon-\mathrm{Fe}_{2} \mathrm{O}_{3}$ подразумевает небольшое искажение окружения атомов $\mathrm{Fe}$, расположенных в октаэдрических $A$ - и $B$-положениях, тогда как существенно меньшее значение QS для секстиплета $C$-подрешетки предполагает высокосимметричное и неискаженное окружение атомов $\mathrm{Fe}$, расположенных в октаэдрических $C$-положениях $\varepsilon-\mathrm{Fe}_{2} \mathrm{O}_{3}$. Основываясь на значении QS для секстиплета $D$-подрешетки, тетраэдрические $D$-узлы $\mathcal{\varepsilon}-\mathrm{Fe}_{2} \mathrm{O}_{3}$ также имеют определенную степень искажения; однако оно не столь выраженное, как для октаэдрических $A$ - и $B$-положений $\varepsilon-\mathrm{Fe}_{2} \mathrm{O}_{3}$ (см. табл. 1 и 2). Поскольку сферически-симметричное распределение электронного заряда для ионов $\mathrm{Fe}^{3+}$ приводит к нулевым орбитальным и диполярным вкладам магнитных сверхтонких полей, то определяемое только отрицательным выражением Ферми общее эффективное магнитное поле, является противоположно ориентированным по отношению к магнитному моменту иона $\mathrm{Fe}^{3+}$. В этом случае сверхтонкое магнитное поле иона $\mathrm{Fe}^{3+}$ в определенной позиции прямо пропорционально намагниченности подрешетки, к которой принадлежит этот ион $\mathrm{Fe}^{3+}$. Значения сверхтонких магнитных полей, соответствующих отдельным спектральным компонентам $\varepsilon-\mathrm{Fe}_{2} \mathrm{O}_{3}$, таким образом, отражают намагниченности конкретных подрешеток при данной температуре.

Для объяснения некоторых различий полученных модельных спектров от наблюдаемых МС отдельных феррооксидов необходимо рассмотреть возможность замещения в феррооксидах ионов железа ионами $\mathrm{Ca}^{2+}$ из НАр. Меньшее значение намагниченности МК HAp/FeOxid, чем в со-осажденных образцах оксидов железа также указывает, что в процессе пиролиза происходит реакция и ионы Са занимают позиции в структуре феррооксидов [26]. Для рассмотрения диффузии ионов Са в кристаллическую структуру шпинели, был рассчитан параметр решетки кристаллитов (FeOxid) оксидов железа в МК HAp/FeOxid и он составил $0.8374 \mathrm{~nm}$. 


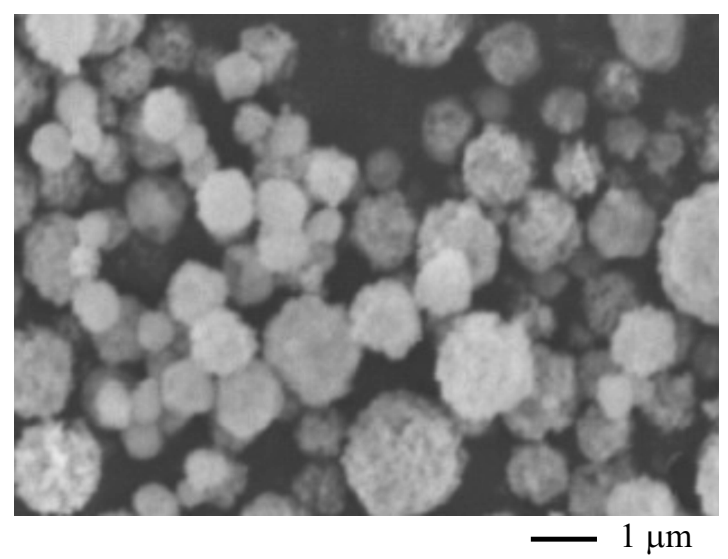

Рис. 3. Фотография композитных магнитных частиц ГАПферрооксиды, полученная с помощью сканирующего электронного микроскопа.

Эта величина несколько больше, чем параметр решетки свободных частиц оксидов железа, как например, в случае $\gamma-\mathrm{Fe}_{2} \mathrm{O}_{3}$ параметр решетки равен $0.8352 \mathrm{~nm}$, что указывает на диффузию ионов Са в структуру $\gamma-\mathrm{Fe}_{2} \mathrm{O}_{3}$, поскольку ионный радиус $\mathrm{Ca}^{2+}$, составляющий $0.100 \mathrm{~nm}$ (в шестикратной координации) превышает радиус иона. Отсюда следует, что величина намагниченности ферритовой компоненты в МК $\mathrm{HAp} / \mathrm{FeOxid}$ меньше, чем у образца, полученного методом со-осаждения, поскольку магнитные ионы $\mathrm{Fe}^{2+}$ в МК HAp/FeOxid замещаются немагнитными $\mathrm{Ca}^{2+}$. Понижение намагниченности при замещении ионов $\mathrm{Fe}$ ионами Са сообщалось в [8-12]. Небольшие изменения структуры магнетита и гексагонального феррита было обнаружено при синтезе в композитах $\mathrm{Hap} / \mathrm{Fe}_{3} \mathrm{O}_{4}$ [7] и $\mathrm{HAp} /$ гексаферрит $\mathrm{Ba}-\mathrm{M}$ [8-10]. Возможно, в МК HAp/FeOxid формируется промежуточный слой между НАр и частицами феррооксида, как это наблюдалось в случае композитов $\mathrm{SrFe}_{12} \mathrm{O}_{19} / \mathrm{La}_{x} \mathrm{Ca}_{1-x} \mathrm{MnO}_{3}$ [39,40], однако, сложность мессбауэровских спектров МК $\mathrm{HAp} / \mathrm{FeOxid}$ не позволяет выделить линии, принадлежащие такому промежуточному слою.

На рис. 3 показаны полученные с помощью СЭМ-фотографии МК НAp-FeOxids. Как видно на рис. 3, композитные частицы имеют шарообразную форму с небольшой ямочкой, что характерно для частиц, синтезированных методом пиролиза ультразвуковой аэрозоли. В случае водного раствора, углубления на частицах формируются осаждением на поверхности из-за высокой скорости сушки аэрозоля [26]. Можно предположить, что углубления на МК, это не покрытая поверхность частицы или слабая агломерация зерен. Как видно на рис. 3, размер МК меняется от 500 до $1000 \mathrm{~nm}$, а средний диаметр составляет $740 \mathrm{~nm}$. Это согласуется с данными, полученными с помощью мессбауэровской спектроскопии, поскольку на мессбауэровских спектрах наблюдаются зеемановские секстиплеты.

При наложении внешнего переменного магнитного поля частотой $370 \mathrm{kHz}$ и напряженностью $1.77 \mathrm{kA} / \mathrm{m}$ на порошковый образец МК весом $3.4 \mathrm{~g}$ (при весе ферритовой компоненты $1.0 \mathrm{~g}$ ) температура образца повышается на $9^{\circ} \mathrm{C}$ за $20 \mathrm{~min}$ и затем стабилизируется [26].

\section{4. Выводы и перспективы}

Исследованы синтезированные методом пиролиза ультразвуковой аэрозоли магнитные композитные частицы гидроксиапатит/феррооксиды (HAp-FeOxids) сферической формы. Установлено, что композит состоит из магнитных частиц феррооксидов, внедренных в структуру гидроксиапатита. Мессбауровские исследования показали, что оксид железа в синтезированных композитах состоит из фаз, принадлежащих феррооксидам: $\gamma-\mathrm{Fe}_{2} \mathrm{O}_{3}$, $\mathrm{Fe}_{3} \mathrm{O}_{4}, \varepsilon-\mathrm{Fe}_{2} \mathrm{O}_{3}$ и $\beta$-FeOOH, причем фаза $\varepsilon-\mathrm{Fe}_{2} \mathrm{O}_{3}$ составляет около $40 \%$. Синтезированные композитные частицы Hap/FeOxids являются биологически совместимыми и обладают большим потенциалом для применений в качестве средств магнитной целенаправленной доставки лекарственных средств и гипертермии. Для повышения концентрации в МK Hap/FeOxid фазы $\varepsilon$ - $\mathrm{Fe}_{2} \mathrm{O}_{3}$, обладающей уникальными свойствами, необходимы дальнейшие исследования с целью поисков оптимальных условий синтеза в композите однофазного $\varepsilon-\mathrm{Fe}_{2} \mathrm{O}_{3}$ с требуемыми размерами и формой и термическим стабилизировать его. Все это позволит создать новый высокоэффективный материал для разнообразных практических применений, в том числе и биомедицинских.

\section{Список литературы}

[1] K. Hayashi, Y. Sato, W. Sakamoto, T. Yogo. ACS Biomat. Sci. Eng. 3, 95 (2017).

[2] Z. Ling-Yun, L. Jia-Yi, O. Wei-Wei, L. Dan-Ye, L. Li, L. Li-Ya, T. Jin-Tian. Chin. Phys. B 22, 108104 (2013).

[3] Magnetic Nanoparticles for Magnetic Hyperthermia and Controlled Drug Delivery. P. Guardia, A. Riedinger, H. Kakwere, F. Gazeau, T. Pellegrino. Pt 6 in Bio- and Bioinspired Nanomaterials / Eds. D. Ruiz-Molina, F. Novio, C. Roscini. Wiley-VCH Verlag GmbH\&Co (2015). 460 p.

[4] K. Chatterjee, S. Sarkar, K.J. Rao, S. Paria. Adv. Colloid Interface Sci. 209, 8 (2014).

[5] M. Kawashita, M. Tanaka, T. Kokubo, Y. Inoue, T. Yao, S. Hamada, T. Shinjo. Biomater. 26, 2231 (2005).

[6] D.-L. Zhao, X.-W. Zeng, Q.-S. Xia, J.-T. Tang, J. Alloy. Compd. 469, 215 (2009).

[7] Н.В. Ткаченко, А.С. Камзин. ФТТ 58, 4, 740 (2016).

[8] Н.В. Ткаченко, Л.П. Ольховик, А.С. Камзин. ФТТ 53, 8 , 1512 (2011).

[9] M.V. Tkachenko, L.P. Ol'khovik, A.C. Камзин, S. Keshri. Письма в ЖТФ 40, 1, 9 (2014).

[10] M.V. Tkachenko, A.S. Kamzin, L.P. Ol'khovik, T.M. Tkachenko, S. Keshri. Solid State Phenomena 215, 480 (2014).

[11] K. Kelm, W. Mader. Z. Anorg. Allg. Chem. 631, 2383 (2005).

[12] А. Ланчок, М. Миглиерини, Я. Когоут. ФММ 109, 562 (2010).

[13] M. Kurmoo, J.L. Rehspringer, A. Hutlova, C. Orle'ans, S. Vilminot, C. Estournes, D. Niznansky. Chem. Mater. 17, 1106 (2005).

[14] S. Sakurai, J. Jin, K. Hashimoto, S. Ohkoshi. J. Phys. Soc. Jpn. 74, 1946 (2005). 
[15] S. Ohkoshi, S. Sakurai, J. Jin, K.J. Hashimoto. J. Appl. Phys. 97, 10K312 (2005).

[16] M. Gich, C. Frontera, A. Roig, E. Taboada, E. Molins, H.R. Rechenberg, J.D. Ardisson, W.A.A. Macedo, C. Ritter, V. Hardy, J. Sort, V. Skumryev, J. Nogue's. Chem. Mater. 18, 3889 (2006).

[17] S.S. Yakushkin, D.A. Balaev, A.A. Dubrovskiy, S.V. Semenov, K. A. Shaikhutdinov, M.A. Kazakova, G.A. Bukhtiyarova, O.N. Martyanov, O.A. Bayukov. J. Supercond. Nov. Magn. 31, 1209 (2018).

[18] J. Tucek, R. Zboril, A. Namai, S.I. Ohkoshi. Chem. Mater. 22, 6483 (2010).

[19] H.L. Liu, C.H. Sonn, J.H. Wu, K.-M. Lee, Y.K. Kim. Biomaterials 29, 4003 (2008).

[20] A. Nadara, A. Mohan Banerjeea, M.R. Paia, S.S. Meena, R.V. Pai, R. Tewarid, S.M. Yusufb, A.K. Tripathia, S.R. Bharadwaj. Appl. Catalysis B 217, 154 (2017).

[21] M.M.A. Nikje, M. Vakili. Current Pharmaceutical Design 21, 5312 (2015).

[22] Н.В. Ткаченко, А.С. Камзин. ФТТ 57, 2, 388 (2015).

[23] Н.В. Ткаченко, А.С. Камзин. ФТТ 58, 8, 1502 (2016).

[24] B. Govindan, B.S. Latha, P. Nagamony, F. Ahmed, M.A. Saifi, A.H. Harrath, S. Alwasel, L. Mansour, E.H. Alsharaeh. Nanomaterials 7, 138 (2017).

[25] F. Foroughi, S.A. Hassanzadeh-Tabrizi, J. Amighian. J. Magn, Magn, Mater. 382, 182 (2015).

[26] A. Inukai, N. Sakamoto, H. Aono, O. Sakurai, K. Shinozaki, H. Suzuki, N. Wakiya. J. Magn. Magn, Mater. 323, 965 (2011).

[27] N. Wakiya, M. Yamasaki, T. Adachi, A. Inukai, N. Sakamoto, D. Fu, O. Sakurai, K. Shinozaki, H. Suzuki. Mater. Sci. Eng. B 173, 195 (2010).

[28] H. Das, N. Debnath, A. Toda, T. Kawaguchi, N. Sakamoto, H. Aono, K. Shinozaki, H. Suzuki, N. Wakiya. Adv. Powd. Tech. 28, 1696 (2017).

[29] N.N. Greenwood, T.C. Gibb. Mossbauer Spectroscopy. Chapman and Hall, Ltd., London (1971).

[30] E. Murad. Phys. Chem. Miner. 23, 248 (1996).

[31] В.Г. Семенов. Программа обработки мессбауэровских спектров МОСФИТ (частное сообщение).

[32] J. Drbohlavova, R. Hrdy, V. Adam, R. Kizek, O. Schneeweiss, J. Hubalek. Sensors 9, 2352 (2009).

[33] A.A. Dubrovskiy, D.A. Balaev, K.A. Shaykhutdinov, O.A. Bayukov, O.N. Pletnev, S.S. Yakushkin, G.A. Bukhtiyarova, O.N. Martyanov. J. Appl. Phys. 118, 213901 (2015).

[34] O. Malina, J. Kaslik, J. Tucek, J. Cuda, I. Medrik, R. Zboril. AIP Conf. Proc. 1622, 89 (2014).

[35] R.M. Cornell, U. Schwertmann. The Iron Oxides: Structure, Properties, Reactions, Occurrences and Uses. Wiley-VCH: Weinheim, Germany (2003).

[36] H. Zeng, J. Li, J.P. Liu, Z.L. Wang, S. Sun. Nature 420, 395 (2002).

[37] J.H. Lee, Y.M. Huh, Y.W. Jun, J.W. Seo, J.T. Jang, H.T. Song, S. Kim, E.J. Cho, H.G. Yoon, J.S. Suh, J. Cheon. Nature Med. 13, 95 (2007).

[38] J. Tucek, R. Zboril, D. Petridis. J. Nanosci. Nanotechnol. 6, 926 (2006).

[39] P. Lampen-Kelley, A.S. Kamzin, K.E. Romachevsky, D.T.M. Hue, H.D. Chinh, H. Srikanth, M.H. Phan. J. All Comp. 636, 323 (2015).

[40] А.С. Камзин, P. Lampen-Kelley, М.H. Phan. ФТT 58, 4, 767 (2016).

Редактор Т.Н. Василевская 